\title{
Cardiac papillary fibroelastomas: A 10-year single-center surgical experience and long-term echocardiographic follow-up study
}

\author{
Martin Koleka, , Jana Dvorackovac , Oldrich Motykad,e, Radim Brat ${ }^{\mathrm{b}, \mathrm{f}}$
}

\begin{abstract}
Aims. Limited contemporary data are available on the clinical and echocardiographic outcomes after surgery for cardiac papillary fibroelastoma (CPF). The aim of this study was to review the clinical manifestations, pathological characteristics, surgical management, and prognoses of patients with histologically verified CPF, who underwent surgery at our cardiac surgery center from 2008 to 2018.

Methods and Results. Twelve patients of median age 62 years (28 to 77 years) were treated. Embolic stroke or transient ischemic attack (five patients, 42\%) were the only CPF clinical manifestations. Eleven (92\%) tumors were localized on the valves, with the aortic valve being the most common tumor site (seven patients; $58 \%$ ). Multiple factor analysis revealed no independent predictor of CPF-related embolization. Simple shave tumor excision was sufficient in most patients (10 patients, $83 \%$ ). No operative or tumor-related late mortality during the median follow-up period of 4.7 years (1.1 to 10.2 years) was recorded. Asymptomatic metachronous valve tumor recurrence (in a location different from that of the original tumor) was revealed in two patients (17\%) by transesophageal echocardiography (TEE), not detected by transthoracic echocardiography (TTE). One of these two patients underwent repeated surgery for CPF but later suffered a recurrent embolic stroke, due to another tumor recurrence.

Conclusion. CPF can be safely and effectively treated surgically. TEE is superior to the TTE option in CPF post-operative recurrence detection. There is a clear need for a prospective study to determine criteria for embolization risk stratification and optimum management in patients with CPF.
\end{abstract}

Key words: cardiac papillary fibroelastoma, embolism, stroke, echocardiography, cardiac surgical procedures

Received: March 22, 2019; Accepted: October 18, 2019; Available online: November 18, 2019

https://doi.org/10.5507/bp.2019.053

(c) 2020 The Authors; https://creativecommons.org/licenses/by/4.0/

${ }^{a}$ Department of Clinic Subjects, Faculty of Medicine, University of Ostrava, Ostrava, Czech Republic

${ }^{b}$ Department of Cardiac Surgery, University Hospital Ostrava, Ostrava, Czech Republic

Institute of Pathology, Faculty of Medicine, University of Ostrava, Ostrava, Czech Republic

${ }^{d}$ Nanotechnology centre, VSB - Technical University of Ostrava, Ostrava, Czech Republic

${ }^{e} E N E T$ centre, VSB - Technical University of Ostrava, Ostrava, Czech Republic

${ }^{f}$ Department of Surgical Studies, Faculty of Medicine, University of Ostrava, Ostrava, Czech Republic

Corresponding author: Martin Kolek, e-mail:martin.kolek@osu.cz

\section{INTRODUCTION}

Primary cardiac tumors are rare, with autopsy studies reporting incidences between $0.0017 \%$ and $0.28 \%$. Seventy-five percent of those are benign ${ }^{1,2}$. The most common benign cardiac tumor in adults is the cardiac papillary fibroelastoma (CPF) (ref., $\left.{ }^{3,4}\right)$. As observed by current reports using echocardiography for clinical detection of the tumor, its occurrence is underestimated in older autopsy studies ${ }^{5,6}$; for example, Tamin et al. ${ }^{4}$ reported the frequency of this tumor at $0.089 \%$ of all patients undergoing echocardiography. The histological description is that of an avascular papillomatous tumor with a single layer of endothelial cells covering the papillary surface ${ }^{7}$ and containing variable amounts of fine elastic fibrils arranged in whorls in a hyaline stroma; surface thrombi may also be present. Typically, it affects cardiac valves, especially the aortic valve. CPF can be a source of non-fatal or fatal thromboembolism or, rarely, interfere mechanically with the valvular function. A complete surgical excision of the tumor is the most effective curative treatment ${ }^{4,8-11}$.

Only limited contemporary data are available on CPF treatment and outcomes, and studies using transesophageal echocardiography (TEE) $\left(\right.$ ref. $\left.^{4,10}\right)$ for follow-up are especially rare. This study retrospectively examines the clinical manifestation, pathology, surgical management, and clinical and echocardiographic outcomes of histologicallyverified cardiac papillary fibroelastomas in patients who underwent surgery at our tertiary cardiac surgery center.

\section{MATERIALS AND METHODS}

\section{Patients and data collection}

The study was approved by The Ethics Committee of the University Hospital Ostrava, Czech Republic, approval No. 821/2017. All patients signed their informed consent and agreed with the publication of the results. 
Patients were identified from our cardiovascular surgery and pathology databases. Clinical histories, echocardiographic reports, operative notes, and histopathological findings were reviewed. Data on symptoms, localization, size, and mobility of CPFs were collected, along with data on potential CPF-caused valvular defects, types of the surgery, complications, and mortality. The principal outcomes were tumor recurrence detected by echocardiography, the clinical recurrence of embolism, and the necessity to re-operate.

During the follow-up, all patients were repeatedly examined by transthoracic echocardiography (TTE) one week, three months and one year after surgery. Subsequent TTEs were performed where the cardiologist considered them beneficial. Where the patient agreed, TEE was performed in addition to the TTE.

\section{Indications for surgery}

Clinical manifestations associated with CPF or asymptomatic mobile tumor were considered primary indications for CPF surgery (any potential additional cardiac pathologies requiring surgical intervention were considered secondary). Where CPF was found incidentally through echocardiography (due to another pathology requiring surgical treatment) or perioperatively, it was removed during the combined surgical procedure. Where asymptomatic non-mobile CPFs that were not referred for a concomitant cardiac surgery were concerned, their surgical removal was the preferred method of treatment.

\section{Surgical techniques}

A complete valve-preserving excision of the tumor (simple shave excision of the root of the pedicle and the endocardium involved) was the surgical technique of choice. Potential defects caused by an extensive excision could be repaired by a direct suture or pericardial patch, should the need arise. Where an associated heart valve disease required surgery to repair or replace the heart valve, it was performed during the same surgery, using standard techniques. All patients were operated on by using median sternotomy and a cardiopulmonary bypass via a transaortic approach or via a left or right atriotomy. In all patients, intraoperative transesophageal echocardiography was performed for assessing the valvular function after tumor excision.

\section{Histopathological analysis}

Histological examination of the obtained tissue samples was performed, using basic hematoxylin/eosin staining as well as the special staining methods acid-Schiff (PAS), Alcian blue, Van Gieson, and elastic stain. In some cases, immunohistochemical analysis (vimentin, desmin, cytokeratin AE1/AE3, CD34, CD31, factor VIII, Ki67, $\mathrm{S}-100$, and calretinin) was performed.

\section{Statistical analysis}

Both the statistical analyses of the data and their graphical presentation were performed in the R environment (R Core Team, 2018, R Foundation for Statistical Computing, Vienna, Austria) (ref. ${ }^{12}$ ). Statistical tests were evaluated at a significance level of $5 \%$. Data were assessed using basic descriptive statistics, including median, arithmetic mean, standard deviation, and frequency tables with absolute and relative numbers. A factorial analysis of mixed data was performed in the $\mathrm{R}$ package "FactoMineR" (ref. ${ }^{13}$ ), and a Kaplan-Meier curve was plotted using the "survival" package ${ }^{14,15}$. A factorial analysis of mixed data allows for the multivariate analysis of datasets containing both numeric (quantitative) and categorical (qualitative) data. It also helps determine vectors of the highest explained variation as well as group the variables according to their association.

\section{RESULTS}

\section{Patients}

From January 2008 to October 2018, 12 patients with CPF were surgically treated at our tertiary cardiac surgery center. Table 1 shows the clinical profile of the study group. The average age of the patients was $62 \pm 14$ years (28-77 years), without a statistically significant difference between sexes ( $58 \%$ of males).

CPF presence was suspected from echocardiography and confirmed histologically after the surgical procedure in all patients. Examples of histopathological and echocardiographic results are shown in the Fig. 1.

CPF was a primary reason for the surgery in eight patients $(67 \%)$. The remaining four patients (33\%) underwent surgery primarily due to symptomatic valve disease. Of these four, CPF was diagnosed preoperatively in two patients; in the remaining two patients, it was found incidentally during surgery. Five patients (42\%) had a personal history of embolism - a recurrent transient ischemic attack in two patients (17\%), and ischemic stroke

Table 1. Clinical features of patients.

\begin{tabular}{lc}
\hline Variable & $\mathrm{n}=12(\%)$ \\
\hline Age (years) & $62 \pm 14$ \\
Male gender & $7(58)$ \\
Indication for surgery & \\
$\quad$ Primary & $8(67)$ \\
Incidental finding & $4(33)$ \\
Presentation & \\
Asymptomatic & $7(58)$ \\
Transient ischemic attack & $2(17)$ \\
Ischemic stroke & $3(25)$ \\
Angina pectoris & 0 \\
Dyspnea & $5(42)$ \\
Palpitations & $2(17)$ \\
Syncope & $1(8)$ \\
Valve dysfunction caused by CPF & 0 \\
Previous cardiac surgery & 0 \\
Concurrent cardiac disease & \\
Coronary artery disease & $3(25)$ \\
Valvular heart disease & $6(50)$ \\
Atrial fibrillation & $3(25)$ \\
\hline
\end{tabular}

$\mathrm{CPF}=$ cardiac papillary fibroelastoma 

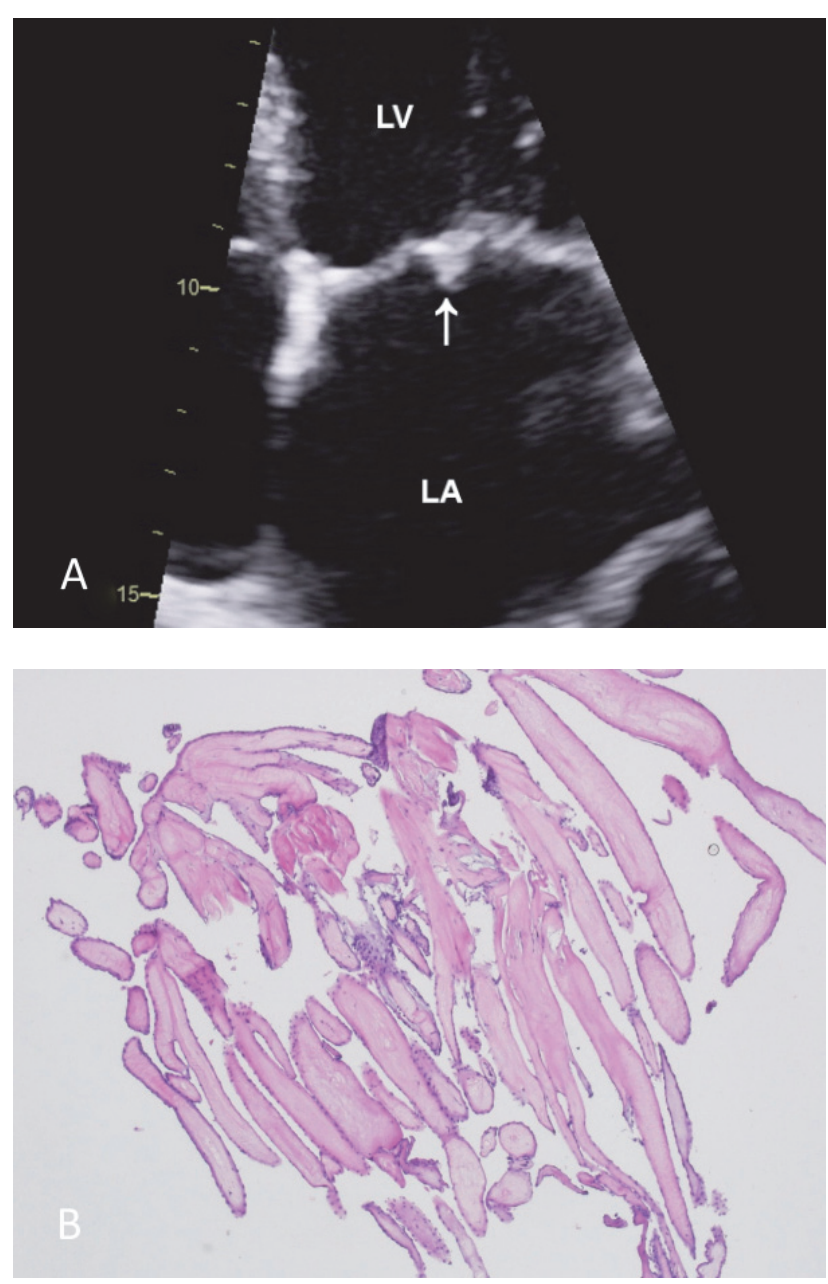

Fig. 1. Papillary fibroelastoma of the mitral valve. (A) Transthoracic echocardiography, apical four-chamber view, tumor on the anterior mitral leaflet (arrow). (B) Microscopic appearance, multiple fronds, hematoxylin/eosin staining, 20x magnification, Olympus DP70. LA = left atrium; LV = left ventricle.

in three patients $(25 \%)$. In the remaining patients, $\mathrm{CPF}$ was asymptomatic. None of the patients had angina pectoris, and eventual dyspnea, palpitations, or syncope were manifestations of coexistent cardiac diseases. CPF did not interfere mechanically with the functions of the affected valves in any of the patients.

In most patients (11 patients, 92\%), CPFs were localized on the valves, as shown in Table 2 . The aortic valve (seven patients; 58\%) was the most common tumor site; in five of these patients, two or three cusps were affected. Altogether, tumorous masses were localized six times on the non-coronary cusp, five times on the left, and three times on the right coronary cusp (perioperative findings). The CPF of the tricuspid valve was localized on its septal leaflet.

The location of the tumor with respect to the valve surfaces is documented in Table 3. The left ventricular-facing, coapting, and aortic-facing surfaces of the noncoronary and left coronary aortic cusp were simultaneously involved in one patient. The left ventricular CPF originated from the posteromedial papillary muscle. No tumors simultaneously affecting multiple valves or heart chambers were found.
Table 2. Location of cardiac papillary fibroelastoma.

\begin{tabular}{|c|c|}
\hline & $\begin{array}{c}\text { Number of patients } \\
n=12(\%)\end{array}$ \\
\hline Valve & $11(92)$ \\
\hline Aortic & $7(58)$ \\
\hline One cusp & 2 \\
\hline Two cusps & 3 \\
\hline Three cusps & 2 \\
\hline Mitral & $3(25)$ \\
\hline One leaflet & 2 \\
\hline Two leaflets & 1 \\
\hline Tricuspid & $1(8)$ \\
\hline One leaflet & 1 \\
\hline Two leaflets & 0 \\
\hline Three leaflets & 0 \\
\hline Cardiac chamber & $1(8)$ \\
\hline Left ventricle & $1(8)$ \\
\hline Right ventricle & 0 \\
\hline Left atrium & 0 \\
\hline \multirow[t]{2}{*}{ Right atrium } & 0 \\
\hline & $\begin{array}{c}\text { Number of lesions } \\
19 \\
\end{array}$ \\
\hline \multicolumn{2}{|l|}{ Valve } \\
\hline Aortic & 14 \\
\hline Noncoronary cusp & 6 \\
\hline Left coronary cusp & 5 \\
\hline Right coronary cusp & 3 \\
\hline Mitral & 4 \\
\hline Anterior leaflet & 2 \\
\hline Posterior leaflet & 2 \\
\hline Tricuspid & 1 \\
\hline Septal leaflet & 1 \\
\hline Anterior leaflet & 0 \\
\hline Posterior leaflet & 0 \\
\hline
\end{tabular}

During the multiple factor analysis, no statistically significant difference between symptomatic and asymptomatic patients was revealed with respect to the tumor location, number of lesions on individual valves, or affected aortic valve surfaces (Fig. 2).

The median diameter of CPF was $5 \times 9 \mathrm{~mm}$, ranging from $2 \times 4 \mathrm{~mm}$ to $20 \times 20 \mathrm{~mm}$. One-way analysis of variance revealed that aortic valve tumors were significantly smaller than the average (mean diameters $3 \times 7 \mathrm{~mm}$ vs. 7 x $9 \mathrm{~mm}, P=0.03$ ). Furthermore, no difference was detected between asymptomatic patients and those after an embolic event (median cross-section diameters 7 × 8 $\mathrm{mm}$ vs. $3 \times 9 \mathrm{~mm}$ ), and therefore no association between tumor size and the development of symptoms could be confirmed (Fig. 2).

Five fibroelastomas (42\%) were mobile spheric or egg-shaped pedunculated, five (42\%) mobile filiform, and two (17\%) sessile. Out of the 10 mobile CPFs, five were asymptomatic and the remaining five symptomatic; both sessile tumors were asymptomatic. The aortic valve tumors were significantly more often mobile $(P=0.02)$. No association between CPF mobility and embolic risk was found (Fig. 2). 
Table 3. Affected valve surface.

\begin{tabular}{ll}
\hline & $\mathrm{n}=9$ \\
\hline Aortic valve & \\
left ventricular-facing & 2 \\
coapting & 1 \\
aortic-facing & 1 \\
all & 1 \\
Mitral valve & \\
atrial-facing & 3 \\
coapting & 0 \\
left ventricular-facing & 0 \\
Tricuspid valve & \\
atrial-facing & 0 \\
coapting & 1 \\
right ventricular-facing & 0 \\
\hline
\end{tabular}

Table 4. Concomitant procedures.

\begin{tabular}{lc}
\hline & $\mathrm{n}=9$ \\
\hline CABG & 2 \\
CABG, MVP, TVP & 1 \\
MVP & 1 \\
MVR & 1 \\
MVP, maze & 1 \\
MVP, TVP, AVR, maze & 1 \\
AVR & 1 \\
maze & 1 \\
\hline
\end{tabular}

$\mathrm{AVR}=$ aortic valve replacement; $\mathrm{CABG}=$ coronary artery bypass grafting; $\mathrm{MVP}=$ mitral valve plasty; $\mathrm{MVR}=$ mitral valve replacement; TVP $=$ tricuspid valve plasty; maze $=$ maze procedure $($ surgical ablation of atrial fibrillation)

\section{Histopathology}

Very small fragments were formed by avascular branching papillary fronds. The stromal tissue was of a mixed fibrous/myxoid character, and the papillary surface was covered by a single layer of flattened polygonal cells. No polysaccharides were detected in PAS staining. In Alcian staining, however, an abundant acidic mucopolysaccharidic and proteoglycan component was found. Van Gieson and elastic staining revealed the organization of collagen and elastic fibers, the latter of which were sparse in places, even completely missing on the apices of the papillae. Avascular fragments were covered with an endothelial layer on the surface. These endothelial cells expressed factor VIII, CD34, CD31, and S-100 proteins, as well as vimentin. Some spindle cells in the deeper layers also expressed S-100 protein. Calretinin and desmin, i.e. markers used for differential diagnosis with myxoma, were negative in the stromal tissue (as typical for CPF), and no Ki67 proliferative activity was detected. In both the stromal cells and the surface endothelial cells, no AE1/ AE3 cytokeratin activity was found.

\section{Surgery}

In most patients ( 10 patients, $83 \%$ ), a simple shave tumor excision without the repair of the valve tumor site was

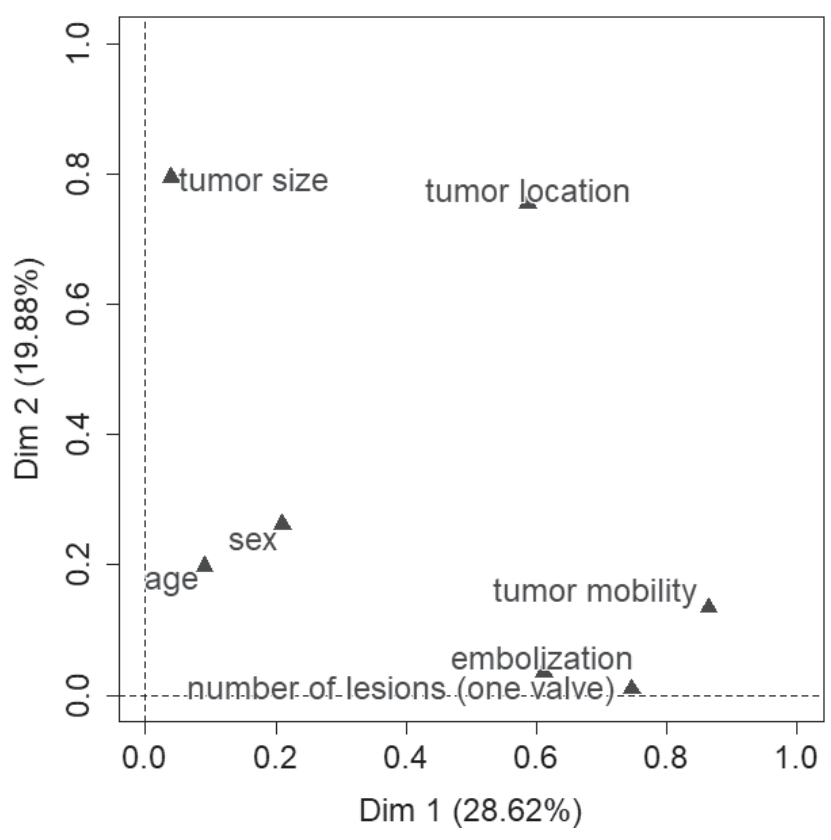

Fig. 2. Risk factors for embolic events. Factorial analysis of mixed data (FAMD), first factor plane. Percentages next to dimension names denote the variability explained alongside the dimension. Proximity of variables indicates their association.

sufficient. In the remaining two patients, shave excision + mitral valve annuloplasty and mitral valve replacement, respectively, were performed; the reason for intervention in these two patients was a symptomatic severe mitral regurgitation, $\mathrm{CPF}$ of the mitral valve was an incidental finding.

Overall, $75 \%$ of patients underwent a combined surgical procedure involving, besides CPF excision, a coronary artery bypass grafting (three patients, $25 \%$ ), a valve repair or replacement (six patients, $50 \%$ ), and a maze procedure (two patients, 17\%), see Table 4 . No patient had a personal history of previous cardiac surgery.

\section{Clinical and echocardiographic outcome}

There was no operative mortality (predicted mortality according to the EuroSCORE II was 3.6\%), see Table 5 . In three patients, however, the following serious postoperative adverse events were recorded (one in each of those patients): Sternal osteomyelitis, cardiac tamponade requiring pericardiocentesis, and ventricular fibrillation, resolved through successful resuscitation and the subsequent secondary preventive implantation of a cardioverterdefibrillator.

The clinical follow-up period ranged from 1.1 to 10.2 years, with a median of 4.7 years. The one-year survival rate was $100 \%$, and the survival rate during the median follow-up time was $92 \%$, see Table 5 . Three patients died over the course of the follow-up, as exemplified in Fig. 3 with a Kaplan-Meier survival curve. Causes of death included pleuropneumonia, generalization of a malign testicular lymphoma, and intracerebral bleeding in a patient with a generalized stomach adenocarcinoma. There was no late mortality related to CPF. 
Four months after surgery, an embolic stroke occurred in one patient with a mechanical mitral valve (surgery performed primarily due to a severe mitral regurgitation, mitral valve CPF was an incidental finding). The embolization occurred at a subtherapeutic international normalized ratio value, without any clear dysfunction of the mechanical prosthesis or CPF recurrence. Following an interventional therapy, no residual neurological deficit was observed. Over the following months, the patient's condition was two times more complicated by a thrombosis of the mitral valve prosthesis. The first one was treated through systemic thrombolysis, during which an embolism in the brain and right kidney occurred. At the second thrombosis of the valve prosthesis, the patient underwent another operation, and the mechanical mitral valve prosthesis was replaced with a bioprosthesis.

Repeated transthoracic echocardiography was performed in all patients during the follow-up. The median TTE follow-up time was 4.2 years ( 0.6 to 9.8 years), and no evidence of any tumor recurrence was found.

In seven patients (58\%), a follow-up TEE was performed as well. The median TEE follow-up time was 1.7 years ( 0.6 to 5.8 years), and no recurrences of cardiac papillary fibroelastoma were observed in five patients $(42 \%$ of the entire group), see Table 5. In the remaining two patients $(17 \%)$ with a follow-up TEE, however, tumor recurrence in a location different from the original one was detected. The first of these two patients originally underwent surgery because of a fibroelastoma originating from the posteromedial papillary muscle of the mitral valve. Six years after the surgery, TEE revealed a $6 \mathrm{~mm}$-long suspected asymptomatic mobile filiform CPF of the non-coronary and right coronary aortic cusps. Due to a relatively high operative risk, the patient did not accept the re-operation and preferred permanent warfarin anticoagulant treatment.

The other patient with complications originally underwent a primary excision of an aortic valve CPF. One year later, TEE revealed a $6 \mathrm{~mm}$-long metachronous asymptomatic mobile filiform CPF of the anterior leaflet of the mitral valve. Repeated surgery-an isolated simple shave excision-was performed with no peri- or postoperative complications. The CPF diagnosis was confirmed histopathologically. A follow-up TEE performed 3.5 months later, however, again detected a newly suspected metachronous asymptomatic mobile filiform tumor of $5-10 \mathrm{~mm}$ on both the aortic and the mitral valves (identical morphology as in the previous detection). Due to the patient's general non-compliance, a decision to treat the patient conservatively with an antiplatelet drug was made; the patient, however, avoided taking the medication. In this patient, the only symptomatic embolic event occurring solely in connection with a CPF diagnosis, namely a recurrent ischemic stroke with a mild residual focal neurologic deficit, was recorded 4.5 months after the second surgery, as shown in Table 5. The recommendation to use anti-aggregation therapy was repeated.

Retrospectively, records of all pre-operative TEE examinations of these two patients were re-examined. No evidence was found of CPF presence in the locations where metachronous CPF recurrences had developed.
Table 5. Outcome.

\begin{tabular}{lc}
\hline & $\mathrm{n}=12(\%)$ \\
\hline Mortality/survival & 0 \\
Operative mortality & $12(100)$ \\
One-year survival & $11(92)$ \\
Survival in median follow-up time & \\
Tumor recurrence & $1(8)$ \\
Clinically & 0 \\
TTE recurrence & $2(17)^{\dagger}$ \\
TEE recurrence & \\
Intervention due to recurrence & $1(8)^{\ddagger}$ \\
Re-operation & $1(8)$ \\
Anticoagulation (warfarin) &
\end{tabular}

$\mathrm{TEE}=$ transesophageal echocardiography $; \mathrm{TTE}=$ transthoracic echocardiography

$\dagger$ metachronous tumors - other than the originally affected site

$\$$ another recurrence of the tumor on both previously affected valves after re-operation, then no surgical/antithrombotic therapy (non-compliance)

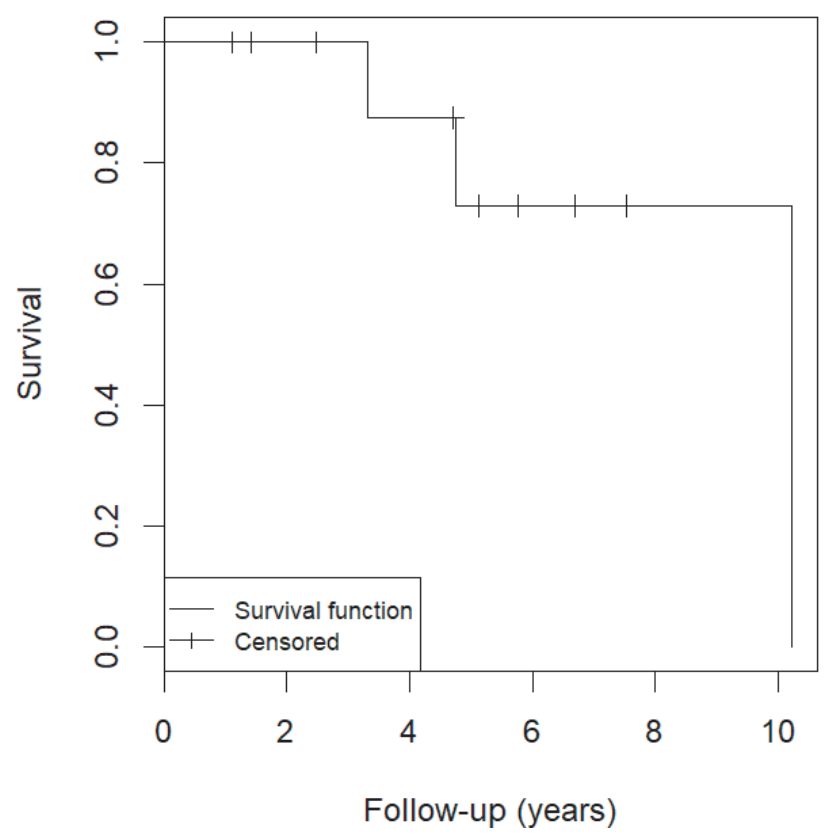

Fig. 3. Kaplan-Meier survival estimate - overall cumulative survival of the entire cohort (the longest follow-up period is terminated by patient's death, hence the drop to 0 ).

Moreover, no abnormalities were apparent on TTE examinations performed between the surgeries and the TEE detection of recurrent tumors, either.

\section{DISCUSSION}

\section{History}

Papillary fibroelastoma was first characterized histologically, histochemically, and by electron microscopy in 1975 by Fishbein et al. ${ }^{16}$. In 1979, Lichtenstein was the first to describe a large incidentally found CPF, resected during the surgical closure of an interventricular septal defect in an adult ${ }^{17}$. The first pre-operative echocardio- 
graphic CPF diagnosis was made by Flotte et al. in 1980 $\left(\right.$ ref. $\left.^{18}\right)$.

\section{Epidemiology}

CPF can occur at any age, but it is nonetheless the most common in the fourth to eighth decades of life. The mean age of detection is approx. 60 years $^{4,5,8-10}$, and in most studies, it has a greater affinity to the male $\operatorname{sex}^{8-10}$. In our patient group, age and sex patterns are consistent with previous studies.

CPFs are either neoplastic processes, hamartomatous lesions, or reactive growths. The CPF etiology remains unknown and no clear risk factor association for the development of this tumor has been discovered to date ${ }^{9}$. The natural history of CPFs has not been defined herein, because no long-term longitudinal follow-up studies utilizing echocardiography have been performed.

\section{Pathology}

According to the comprehensive analysis of 725 cases of CPF, published by Gowda et al. $^{8}$, the valvular surface was the predominant location of the tumor $(84 \%)$. The aortic valve was the most commonly involved (44\%), followed by the mitral valve (35\%). The left ventricle was the predominant non-valvular site of tumor origin. Multiple tumors on a single or multiple location were diagnosed in 39 patients from the abovementioned patient group. Tumor size ranged from 2 to $70 \mathrm{~mm}$, typically around 10 $\mathrm{mm}$ in their greatest dimension.

The pathological-anatomical characteristics of our patient group are similar to those reported in other studies ${ }^{4,9,10}$. In the study by Tamin et al. ${ }^{4}, \mathrm{CPFs}$ in patients presenting with a neurological event resulting from $\mathrm{CPF}$ were significantly smaller than CPFs in those who presented with other indications. Most tumors in our patient group were mobile (84\%), which is almost twice as many as in the study by Sun et al. ${ }^{5}$, who only reported $44 \%$ of mobile CPFs. There is no agreement among studies on the involved valvular surfaces: While Sun et al. ${ }^{5}$ reported that most tumors presented on the aortic side of the valve, Ngaage et al. ${ }^{9}$ found most tumorous masses on the ventricular surfaces of aortic cusps and mitral leaflets, and in the study by Tamin et al. ${ }^{4}$, an approximately equal proportion of CPFs arose from the aortic and ventricular surfaces of the aortic valve. Our results, as far as the aortic valve is concerned, are in agreement with this last mentioned study.

Tumor size/volume, the site of left heart tumors, and the valvular surface involved are not determinants of systemic thromboembolism ${ }^{4,8-10}$. According to Gowda et al. ${ }^{8}$, the only independent predictor of CPF-related death or nonfatal embolization was tumor mobility. In contrast, in the study by Tamin et al. ${ }^{4}$, mobility was not significantly associated with an occurrence of cerebrovascular accidents, possibly due to a smaller group size. In our relatively small patient group, there was no difference in the pathological-anatomical characteristics of CPFs associated or disassociated with embolism.

\section{Clinical diagnostics}

$\mathrm{CPF}$ is increasingly diagnosed clinically, i.e. ante-mortem. The rate of underdiagnosis, however, is still relatively high, because CPFs are often asymptomatic ${ }^{3}$. They are often diagnosed incidentally at the time of echocardiography, computed tomography, cardiac surgery, or autopsy. There is a wide range of possible clinical manifestations of left heart CPFs, the most common of which is an embolic transient ischemic attack or stroke, especially in patients with mitral valve tumors. Aortic valve tumors are more commonly manifested by sudden death or myocardial infarction ${ }^{8,9}$. In our study, embolic neurological events were the only manifestation in patients undergoing surgery primarily for this tumor. Right heart CPF can also manifest through a paradoxical systemic embolism in the presence of a patent foramen ovale ${ }^{9,19}$. Rarely, CPF may cause a valve stenosis or regurgitation, due to an interference with the valve closure. None of these occurred in our patient group.

The imaging method of choice for CPF detection is echocardiography, with TEE allowing a more accurate assessment than TTE. In the study by Tamin et al. ${ }^{4}$, both TTE and TEE revealed the tumor in $51 \%$ of patients, while in $33 \%$ of patients, the tumor could be detected only by TEE. A small, mobile, pedunculated or sessile valvular, or endocardial masses, fluttering or prolapsing into the cardiac chambers during systole or diastole, are observed. There are, however, no specific echocardiographic features allowing a definite diagnosis of CPF. Fibroelastoma should be generally considered if a mobile echogenic structure with a speckled appearance, echolucencies, and a stippled pattern near the edges is detected ${ }^{6}$. Threedimensional echocardiography has an incremental value over two-dimensional echocardiography in the assessment of these tumors, since it allows for acquiring true CPF dimensions and offers additional real-time information on its shape, mobility and relationship to other structures ${ }^{20-22}$. In all of our patients, the presence of CPF was suspected solely on the basis of two-dimensional TEE, and no other imaging methods were used.

\section{Treatment}

The treatment of choice for both symptomatic and asymptomatic mobile CPF is a surgical excision including the full thickness of the endocardium involved. A potential resulting defect is either sutured directly or a pericardial patch closure is performed. A complete surgical excision has a curative effect with a high probability of preserving the affected valve $e^{4,8,23}$. Where the valve is gravely damaged, its repair or replacement are necessary.

In the often quoted study of Gowda et al. ${ }^{8}$, a surgical CPF excision was carried out in 425 patients, of which the valve repair was required in $9 \%$ and a valve replacement in $10 \%$. In a study by Ngaage et al. ${ }^{9}$, comprising 88 patients, CPF was a primary indication for surgery in 53\% of patients and an incidental finding in $47 \%$ of patients. Shave excision was sufficient in most patients $(83 \%)$, eight patients $(9 \%)$ underwent an excision with a valve repair and five $(6 \%)$ had valve replacements. Of these five 
valve replacements, two were due to a concurrent degenerative valve disease. Concomitant procedures included the repair or replacement of another valve (32\%), coronary artery bypass grafting (28\%), and septal myectomy (19\%). In the study by Tamin et al. ${ }^{4}$, histopathologically confirmed CPF was surgically removed in 185 patients; in 94 patients (51\%), CPF was the primary reason for the surgery, while in 91 patients (49\%), the surgery was due to another problem. In valvular CPFs removed during primary surgery, the valve was preserved in 92 patients (98\%). In our patient group, CPF excision was combined with another intervention in a majority of patients. A simple shave tumor excision with preservation of the valve, as in the abovementioned studies, was sufficient in most patients.

Out of 25 patients in the study by Gowda et al. ${ }^{8}$ who did not undergo surgical excision of CPF, 12 patients succumbed to a CPF-related death and four patients suffered a nonfatal embolization. Hence, the authors concluded that symptomatic patients who, for any reasons, do not undergo surgery should be treated with long-term oral anticoagulation; it is, however, necessary to point out here that its efficacy has not been confirmed in any randomized controlled study.

The study by Tamin et al. ${ }^{4}$ includes a relatively large group of 326 patients in whom CPF was echocardiographically suspected but no surgical removal was performed. In these patients, rates of cerebrovascular accident and mortality were increased. The risk of stroke in unoperated CPF was $6 \%$ and $13 \%$ at one and five years, respectively (which was higher than age- and sex-matched rates), while in patients in whom surgery was performed primarily due to $\mathrm{CPF}$, the reported risks were $0 \%$ and $5 \%$ at one and five years, respectively. There was no significant difference in the occurrence of subsequent stroke between patients treated with warfarin or antiplatelet drugs versus those not taking medication. The authors nevertheless concluded that the study may not have the statistical power to evaluate the benefit of antithrombotic therapy.

The management of asymptomatic non-mobile lesions is a controversial topic ${ }^{23}$. An excision of asymptomatic CPF is recommended in patients requiring concomitant cardiac surgery, as it can be achieved with acceptable results $^{4}$. No data, however, are available on the management and therapy of patients with asymptomatic non-mobile CPF who have not been referred for concomitant cardiac surgery. According to Gowda et al. ${ }^{8}$, these patients should be followed up clinically and echocardiographically, since the development of symptoms or the tumor turning mobile is an indication for surgery. No consistent and validated method for the embolism risk prediction is available, though, and so for this reason, other authors recommend an aggressive surgical approach, i.e. performing the surgical removal of CPF in all patients with an acceptable surgical risk $^{4,9,24}$. We believe that it is necessary to perform a randomized trial aimed at developing criteria for risk stratification and preparing guidelines for the management of patients with asymptomatic CPF. Due to the rarity of this disease, however, such a trial would have to be multicentric.

\section{Outcome}

All published studies agree on zero ${ }^{11,25,26}$ or very low (approx. 1\%) (ref. ${ }^{4,9}$ ) in-hospital/30-day mortality in patients undergoing CPF excision.

The long-term prognosis following a surgery for $\mathrm{CPF}$ is good as well. No recurrence of the tumor was reported in most (predominantly older) studies ${ }^{5,8,9,11}$. More recently published papers ${ }^{4,10}$ report very low recurrence rates. This increased capture of (predominantly asymptomatic) recurrences in more recent studies is probably associated with an increased use of echocardiography during the followup. New CPFs may arise at or near the site of a previous resection, due to an endocardial injury; an incomplete resection can be another possible cause of the recurrence.

Anastacio et al. ${ }^{10}$ published their experiences of surgery for CPF in 23 patients (with 29 lesions). After a tumor excision, the mean follow-up was 37 months (range, one month - 10 years). In 18 patients, a postoperative TTE was performed, and in one patient, TEE was performed. A metachronous lesion on the aortic valve was detected in one patient three months after the initial surgical intervention (which was performed primarily due to a lesion on the pulmonary valve and another lesion in the right atrium); the diagnosis was histologically confirmed. Echocardiography was carried out in $66 \%$ of patients at a median of 2.9 years (IQR: 0.9 to 5.3 years) in the already cited paper by Tamin et al. ${ }^{4}$, in which the post-operative recurrence of CPF was $1.6 \%$ (three out of 185 patients). In our group, an asymptomatic metachronous tumor recurrence was revealed in two patients $(17 \%)$ by TEE, while TTE did not reveal any recurrence. In both patients, the tumor developed in a different location than the primary occurrence. In one of these patients, re-operation was successfully performed; however, new asymptomatic recurrences were detected by TEE on both previously affected valves. As expected, TEE is significantly more sensitive than TTE where the detection of recurrent CPFs is concerned. A standard practice of using TEE for follow-up in patients after CPF excision-in our opinion-therefore deserves a thorough discussion.

No tumor-related late morbidity or mortality during the follow-up was reported by Sun $^{5}$ or Ngaage 9 . Ten cerebrovascular accidents (in a group of 185 patients - after both primary or concomitant surgery) were recorded during follow-up by Tamin et al. ${ }^{4}$, with the overall risk of stroke at $2 \%$ and $8 \%$ at one and five years, respectively. In our study group, one embolic stroke associated with CPF recurrence was registered in the abovementioned non-compliant patient.

\section{CONCLUSION}

We present a single cardiac surgery center experience with the surgical treatment of CPF in a small group of patients, including long-term follow-up using echocardiography. No independent predictor of CPF-related nonfatal embolization was identified when using multiple factor analysis. CPF can be safely and effectively treated surgically, and for our patients, a simple shave tumor excision 
was sufficient in almost all cases. There was no operative mortality and no tumor-related late mortality during the long-term clinical follow-up. Transesophageal echocardiography was superior to transthoracic echocardiography in the detection of asymptomatic metachronous tumor recurrence in two patients (in a location different from that of the original tumor). One of these two patients underwent repeated surgery for CPF but later suffered a recurrent embolic stroke, caused by newly recurrent metachronous tumors. The retrospective design and small number of patients were the most significant limitations of this study.

There is a clear need for a prospective study or studies aiming at determining criteria for embolization risk stratification as well as optimum management strategies, in particular in patients with an asymptomatic non-mobile CPF. Based on the data currently available, surgical excision should be considered in patients with a low surgical risk and left-sided CPF regardless of tumor size, mobility, or location. Such an aggressive surgical approach is supported by a high likelihood of preserving the affected valve, a very low postoperative recurrence rate, and a reduction in the risk of CPF-related systemic embolism. Of course, adequate surgical expertise is a must. Further research is needed to evaluate the effectiveness of antiplatelet or anticoagulation therapy, especially in patients who are not candidates for surgical intervention or who decline surgical intervention. Due to the possible presence of thrombotic mass on the tumor surface, antithrombotic therapy should be considered.

Author contributions: MK: study design, data collection, analysis and interpretation, statistical analysis, manuscript preparation, literature search; JD: data collection, manuscript preparation, literature search; OM: statistical analysis; RB: manuscript revision.

Conflict of interest statement: The authors state that there are no conflicts of interest regarding the publication of this article.

\section{REFERENCES}

1. McAllister HA, Fenoglio JJ. Tumors of the cardiovascular system. In Hartmann WH, Cowan WR, eds. Atlas of Tumor Pathology. Second Series, Fascicle 15. Washington, D.C.: Armed Forces Institute of Pathology; 1978. p. 1-3.

2. Reynen K. Frequency of primary tumors of the heart. Am J Cardiol 1996;77(1):107.

3. Edwards FH, Hale D, Cohen A, Thompson L, Pezzella AT, Virmani R. Primary cardiac valve tumors. Ann Thorac Surg 1991;52(5):1127-31.

4. Tamin SS, Maleszewski JJ, Scott CG, Khan SK, Edwards WD, Bruce CJ, Oh JK, Pellikka PA, Klarich KW. Prognostic and Bioepidemiologic Implications of Papillary Fibroelastomas. J Am Coll Cardiol 2015;65(22):2420-9. doi: 10.1016/j.jacc.2015.03.569

5. Sun JP, Asher CR, Yang XS, Cheng GG, Scalia GM, Massed AG, Griffin BP, Ratliff NB, Stewart WJ, Thomas JD. Clinical and echocardiographic characteristics of papillary fibroelastomas: a retrospective and prospective study in 162 patients. Circulation 2001;103(22):2687-93.
6. Klarich K, Enriquez-Sarano M, Gura G, Edwards W, Tajik A, Seward J. Papillary fibroelastoma: echocardiographic characteristics for diagnosis and pathologic correlation. J Am Coll Cardiol 1997;30(3):784-90.

7. Burke A, Virmani R. Papillary fibroelastoma: tumors of the heart and great vessels. AFIP Atlas of Tumor Pathology 1996;16:47-54.

8. Gowda RM, Khan IA, Nair CK, Mehta NJ, Vasavada BC, Sacchi TJ. Cardiac papillary fibroelastoma: a comprehensive analysis of 725 cases. Am Heart J 2003;146(3):404-10. doi: 10.1016/S00028703(03)00249-7

9. Ngaage DL, Mullany CJ, Daly RC, Dearani JA, Edwards WD, Tazelaar HD, McGregor CG, Orszulak TA, Puga FJ, Schaff HV, Sundt TM, Zehr KJ. Surgical treatment of cardiac papillary fibroelastoma: a single center experience with eighty-eight patients. Ann Thorac Surg 2005;80(5):1712-8. doi: 10.1016/j.athoracsur.2005.04.030

10. Anastacio MM, Moon MR, Damiano RJ, Pasque MK, Maniar HS, Lawton JS. Surgical experience with cardiac papillary fibroelastoma over a 15-year period. Ann Thorac Surg 2012;94(2):537-41. doi: 10.1016/j.athoracsur.2012.04.006

11. Ikegami H, Andrei AC, Li Z, McCarthy PM, Malaisrie SC. Papillary fibroelastoma of the aortic valve: analysis of 21 cases, including a presentation with cardiac arrest. Texas Heart Institute journal 2015;42(2):131-5. doi: 10.14503/THIJ-14-4262

12. R Core Team (2018). R: A language and environment for statistical computing. R Foundation for Statistical Computing, Vienna, Austria. Available from: https://www.R-project.org/ [Accessed: October 10, 2019].

13. Le S, Josse J, Husson F. FactoMineR: An R Package for Multivariate Analysis. Journal of Statistical Software 2008;25(1):1-8. doi: 10.18637/ jss.v025.i01

14. Therneau TM, Grambsch PM. Modeling Survival Data: Extending the Cox Model. Springer-Verlag, New York, 2000. ISBN 0-387-98784-3.

15. Therneau TM (2015). A Package for Survival Analysis in S. version 2.38. Available from: https://CRAN.R-project.org/package=survival [Accessed: October 10, 2019].

16. Fishbein MC, Ferrans VJ, Roberts WC. Endocardial papillary elastofibromas. Histologic, histochemical, and electron microscopical findings. Arch Pathol (Chic) 1975;99(6):335-41.

17. Lichtenstein HL, Lee JC, Stewart S. Papillary tumor of the heart: incidental finding at surgery. Hum Pathol 1979;10(4):473-5.

18. Flotte T, Pinar H, Feiner H. Papillary elastofibroma of the left ventricular septum. Am J Surg Pathol 1980;4(6):585-8.

19. Vandergoten $P$, Dendale $P$, Geukens R, Benit E, Vanbockrijk M, Mees $U$, Hendrikx M. Right auricular fibroelastoma and paradoxical embolic stroke. Acta Cardiol 1999;54(1):49-50.

20. Nanda N, Rahman S, Khatri G. Incremental value of three-dimensional echocardiography over transesophageal multiplane two-dimensional echocardiography in qualitative and quantitative assessment of cardiac masses and defects. Echocardiography 1995;12:619-28.

21. Asch FM, Bieganski SP, Panza JA, Weissman NJ. Real-time 3-dimensional echocardiography evaluation of intracardiac masses. Echocardiography 2006;23(3):218-24. doi: 10.1111/j.15408175.2006.00196.x

22. Plana JC. Added value of real-time three-dimensional echocardiography in assessing cardiac masses. Curr Cardiol Rep 2009;11(3):205-9.

23. Yong MS, Smail H, Saxena P. Management of incidental papillary fibroelastoma: An update. Int J Cardiol 2016;215:338-9. doi: 10.1016/j. ijcard.2016.04.113

24. Minatoya K, Okabayashi H, Yokota T, Hoover E. Cardiac papillary fibroelastomas: rationale for excision. Ann Thorac Surg 1996;62(5):151921.

25. Dell'amore A, Albertini A, Lamarra M. Twenty years experience in oncologic surgery for primary cardiac tumors. G Chir 2013;34(4):10611.

26. Mkalaluh S, Szczechowicz M, Torabi S, Dib B, Sabashnikov A, Mashhour A, Karck M, Weymann A. Surgery for Cardiac Papillary Fibroelastoma: A 12-Year Single Institution Experience. Med Sci Monit Basic Res 2017;23:258-63. 